\title{
Design of a Normalized Delayless LMS Adaptive Subband Digital Filter
}

Shing Tenqchen*, Ming-Chang Sun, and Wu-Shiung Feng+

* Lab 353. Department of Electrical Engineering, National Taiwan University, Taipei, Taiwan. ROC

Also with Chunghwa Telecom Telecommunication Labs., 12, Lane 551, Sec. 5 ,

Min-Tsu Rd., Yang-Mei Zien, Tao-Yuan County, Taiwan 326, ROC

Labs 353, Department of Electrical Engineering, National Taiwan University, Taipei, Taiwan. ROC

+ Department of Êlectronics Engineering, Chang Gung University, Taoyuan, Taiwan R, O. C.

Email: d6921028@ms.cc.ntu.edu.tw and stclams.chul.com.tw

\begin{abstract}
A new archilecture is proposed for the Normalized Delayless LMS (NDLMS) adaptive subband filter. The proposed architecture is based on the hardware-efficient pipelined architecture for the LMS adaptive filter and subband methodology. and it can achieve good convergence characteristics, short latency and high throughput simultaneously without adaptation delays. In addition, it remains the advantage of the NLMS i.e., the step size assures that the convergence rate is determined automatically. Computer simulation results that confirm that the proposed architecture achieves satisfied convergence rate identical to those of the NLMS in frequency-domain.
\end{abstract}

\section{INTRODUCTION}

In this paper, we propose a new approach for the architecture adaptive digital filters (ADFs) based on the normalized least mean square (normalized LMS: NLMS) algorithm in frequencydomain. It maintains the advantage of the NLMS, which is that the value of step-size parameter is determined automatically, thereby ensuring convergence.

Recently, the conversion algorithm from DLMS to LMS [1] is deserved much attention due to the improvement of convergence by adding

- This work was supported by National Science Council Under NSC: 88-2216-E-002-018 correctional term to the DLMS. Although this action improves the convergence, it requires a large amount of calculations to be implemented. Pipelined processing of gradient-type ADFs has been considered [2-5] as a possibly way to meet the requirements of processing signals at very high speed. The new approach for LMS algorithm was classified by Shynk [6] in frequency-domain. He has pointed out the frequency domain has primary two advantages compared to time-domain implementations. The first is the potential large saving in the computational complexity. Second, it is the DFT and filter bank structures to generate signals that are approximately uncorrelated (orthogonal) without the effect of delay. It is the motivation for us to implement it to transform NDLMS to NLMS to save the calculation time in frequency-domain.

This paper is organized as following. Section 2 is the problem formulation. Then, we give the derivation of the proposed architecture in Section 3. In Sect. 4, simulation results of the characteristics of the proposed architecture with the conventional ones are shown. It is shown, in Section 5, to have good convergence characteristics, short latency and high throughput, which is identical to the NLMS in frequency-domain. 


\section{Problem Formulation \\ NDLMS Algorithm}

Let us consider the least mean square adaptive filter with delay problem. The NDLMS has $D$ $(0 \leq D \leq N)$ units of delay in the error feedback path. An $N$ th tap standard time-domain NDLMS algorithm has the following nonblock coefficient (weight) update equations:

$$
\begin{aligned}
& w(n+1)=w(n)+\frac{\alpha}{1+\gamma\|x(n-k)\|^{2}} \times x(n-D) \times e(n-D),(1 \mathrm{a}) \\
& y(n)=x^{T}(n) w(n), \quad(1 \mathrm{~b}) \\
& e(n-D)=d(n-D)-w^{T}(n-D) x(n-D) \\
& =d(n-D)-w^{T}(n-D+\delta) x(n-D) \\
& +\mu \sum_{i=0}^{D-1} e(n-2 D+i) x^{T}(n-2 D+i) x(n-D)
\end{aligned}
$$

where $d(n)$ and $y(n)$ denote the desired signal, and output signal, respectively. $\alpha$ and $\gamma$ are some constants chosen by user to get the robust step-size used for adaptation of the weight vector.

We can rewrite Eq. (1c) as

$$
\begin{aligned}
& \varepsilon(n-D)=e(n-D)-\Lambda(n), \\
& \varepsilon(n-D)=d(n-D)-w^{T}(n-D+\delta) x(n-D)-\Lambda(n), \\
& \Lambda(n)=\mu \sum_{i=1}^{D-1} e(n-2 D+i) x(n-2 D+i)^{T} x(n-D),
\end{aligned}
$$

where $\Lambda(n)$ is the correctional term [1]. By adding the correctional term to the error signal of the $N D L M S$, we can decrease the time lag between $w(n)$ in Eq. (1a).

Consider a block recursion in (1) of the weights from time $n$ to time $n+L$ based on the $L$ data samples accumulated. Without loss of generality, we can substitute $n=k L$ where $n$ is an integer multiple of $k$. The block gradient estimate is given by the following summation:

$$
\begin{aligned}
& W(k+1)=W(k)+2 \mu \chi^{\prime \prime}(k-D) E(k-D), \\
& y(k)=\sum_{m=0}^{L-1} \chi_{m}(k) W(k L+m-D),
\end{aligned}
$$

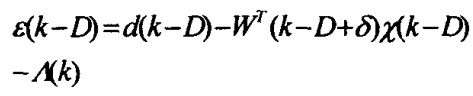

$$
\Lambda(\mathrm{k})=\mu \sum_{i=0}^{D-1} E(k-2 D+i) \chi(k-2 D+i)^{H} \chi(k-D)
$$

where $\chi_{m}^{T}(k)$ is the $m$-th row of $\chi(k)=F^{-1} X(k) F$ and $F$ is a full matrix (but with rank $\leq 2 N$ DFT matrix. It is also possible to rewrite the output vector in (3b) using similar matrix representation:

$y(k)=k F^{-1} Y(k)$,

where $k$ is the following constraint matrix:

$k=\left[\begin{array}{ll}0_{N} & I_{N}\end{array}\right]$

The FFT algorithm should be used to compute the error vector

$E(k)=F k^{T} e(k)$.

The block NDLMS (BNDLMS) algorithm in (3) essentially minimizes the same $M S E$ performance function as the nonblock $L M S$ algorithm in (1). For wide-sense stationary signals, the steady-state weight vector (Wiener solution), misadjustment, and time constants of the BNDLMS algorithm are identical to those of the standard NDLMS algorithm. The main difference is that the maximum value of the step size such that the algorithm is stable is now scaled down by a factor of $L[6],[7]$.

The frequency-domain weight vector is similar to the block update in (3). Because DFT computations inherently perform a circular convolution [6], the adaptive filters generally require data constraints in order to implement the desired linear convolution. Analogous to (1) and (2), define the frequencydomain weight vector as

$W(k)=\left[w_{0}(k), \ldots, w_{M-I}(k)\right]^{\mathrm{T}}$.

and the input signal matrix as

$X(k)=\operatorname{diag}\left\{x_{0}(k), \ldots, x_{M-1}(k)\right\}$,

where $\operatorname{diag}\{$.$\} is an operator that forms a diagonal$ matrix. The number of elements, $M$, depends on the frequency-domain analog filter (FDAF) 
configuration (usually $M=N$ or $M=2 N$ ). With these definitions, the general form of the FDAF algorithms can be expressed as

$W(k+1)=W(k)+2 \mu(k) \chi^{H}(k-D) E(k-D)$

Where the superscript $H$ denotes complex conjugate transpose. Generally, each step size is varied according to the signal power in that frequency bin. We could have $\mu_{\mathrm{m}}(k)=\alpha /\left(1+\gamma|| X_{m}(k-\right.$ $D) \|^{2}$ ) where $m$ is a fixed scalar and $P_{m}(k)$ is an estimate of the signal power in the $m^{\text {th }}$ bin, which might be computed as

$P_{m}(k)=\lambda \operatorname{Pm}(\mathrm{k}-1)+\alpha\left|X_{m}(k)\right|^{2}, \mathrm{~m}=0, \ldots, \mathrm{N}-1$

where $\lambda=1-\alpha$ is a forgetting factor. If the data are statistically stationary, the step-size matrix may be fixed such that $\mu_{\mathrm{m}}(k)=\mu_{\mathrm{m}}$, or each step-size may even be identical. The complete algorithm, which was first derived by Dentino et al. [8], is summarized in Table 1.

Table 1.

FDAF Algorithm Based on Circular Convolution Initialization:

$W(0)=[0, \ldots, 0]^{\mathrm{T}} ; P_{m}(0)=\delta_{\mathrm{m}}, m=0, \ldots, \mathrm{N}-1$.

Matrix Definition:

$\mathrm{F}=N \mathrm{x} N D F T$ matrix

For each new block of $\mathbf{N}$ input samples:

$X(k)=\operatorname{diag}\left\{F\{x(k N), \ldots, x(k N+N-1)\}^{\mathrm{T}}\right\}$

$D(\mathrm{k})=F d(k)$

$Y(k)=X(k) W(k)$

$E(k)=D(k)-Y(k)$

$P_{m}(k)=\lambda P_{m}(k-1)+\alpha\left|X_{m}(k)\right|^{2}, m=0, \ldots, N-1$.

$\mu(k)=\mu \operatorname{diag}\left\{P_{0}^{-1}(k), \cdots, P_{N^{\prime}-1}^{-1}(k)\right\}$

$W(k+1)=W(k)+2 \mu(k) \chi^{H}(k) E(k)$

\section{Transform NDLMS to NLMS without Delay}

Effect in Frequency-Domain.

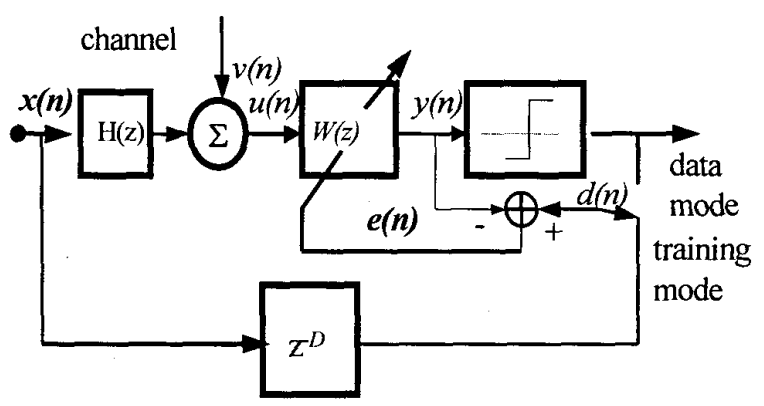

Fig. 1 Adaptive linear equalizer with training.

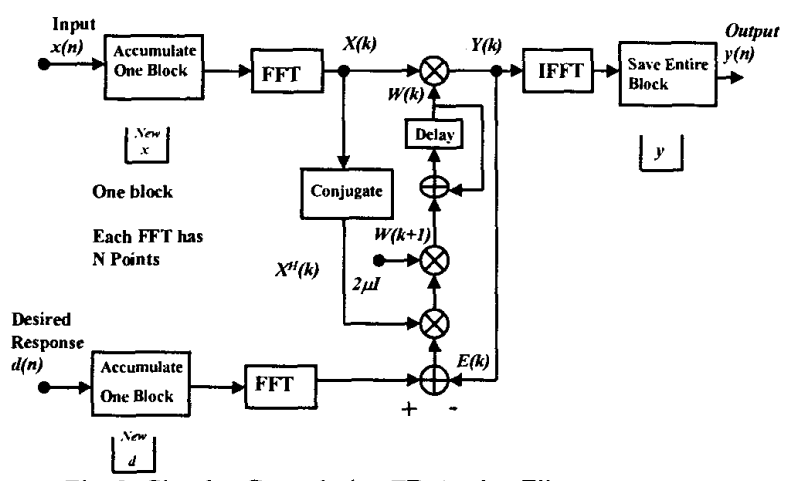

Fig. 2. Circular-Convolution FD Analog Filter

This paper is focused on the NDLMS algorithm transformed to $N L M S$ which has a linear error function, i.e. $e(k)=d(k)-y(k)$. As such, the structure of adaptive linear equalizer described in Fig. 1 [6] are often equivalent and it is usually straight forward to develop $F D A F$ algorithms where the error is computed in either the time or frequency domains. The $F D A F$ algorithm based on circular convolution is plotted in Fig. 2 [6].

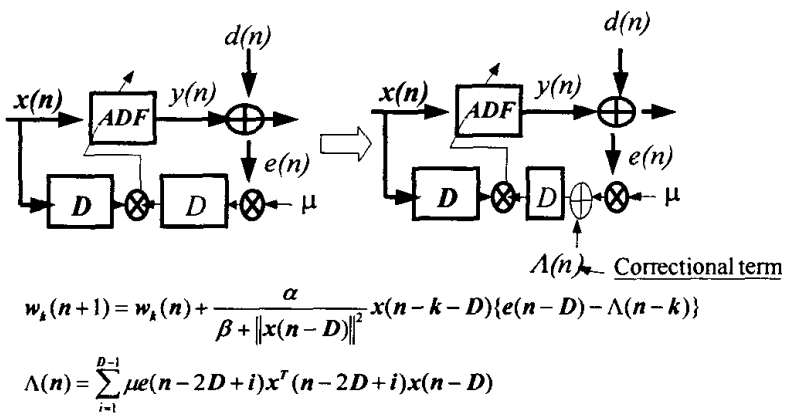

Fig. 3a Transform from DLMS to LMS in Time-Domain by correctional term. 
Figure 3a shows that the block diagram of the modified DLMS architecture is transformed from $D L M S$ to $L M S$ by correctional term in [5]. Note that when $\delta=0$, this algorithm is reduced to the $D L M S$. When $\delta=D$, this algorithm is reduced to $L M S$ in equation (3c). Analogously, by observing the structure of Fig. 2 in frequency domain, the inserting correctional term in the feedback path section will create the same effect with that of in time domain. The equation-error formulation is related to adaptive FIR filtering because there is no output feedback in the filter. As a result, the convergence properties of this adaptive filter are well understood and readily predicted. The main difference is that the desired response is filtered by a set of "feedback" coefficients, which is analogous to the set of feedforward coefficients that weight the input signal [6]. All of the previous FDAF configurations can be modified to accommodate the equation-error formulation, as demonstrated here for the overlap-save linear convolution method.

Fig. $3 b$ and $3 c$ show the structure of the signal flow graph $(S F G)$ of the K-N-K model [5]. The K$\mathrm{N}-\mathrm{K}$ model has proven a new pipelined architecture for $L M S$ adaptive filter, which performs good convergence characteristics, short latency and high throughput characteristics. The pipelined correctional DLMS is called PcDLMS for short writing.

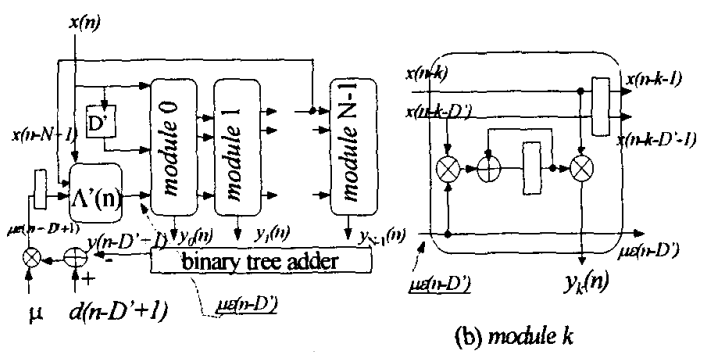

(a) SFG of Pipelined cDLMS

Fig. 3b Signal Flow Graph (SGF) of K-N-K's PcDLMS.

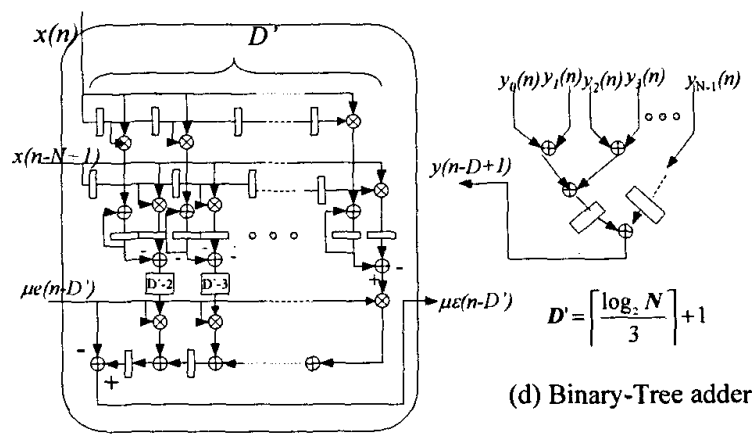

(C) Correctional term $\Lambda^{\prime}(n)$

Fig. 3c SFG of K-N-K's PcDLMS

Inherited from this good pipelined structure, we extend it to the frequency-domain from Fig. 2 to Fig. $3 d$.

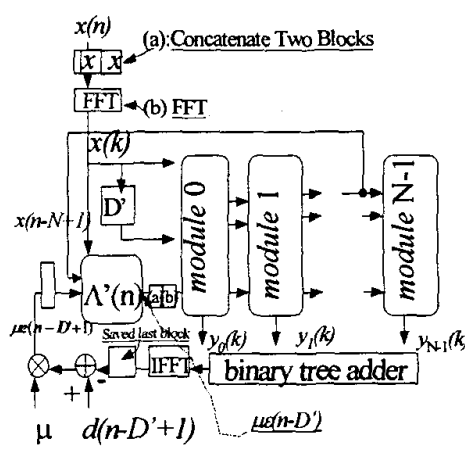

(a) SFG of PcDLMS

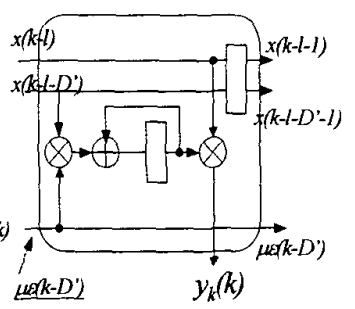

(b) mocule $k$
Fig. 3d. The proposed SFG of modified K-N-K's PcDLMS.

It shall be noticed that the proposed $S F G$ of modified K-N-K's PcDLMS is built with the same structure of correctional term and binary-tree adder as those of in Fig. 3c.

\section{Simulation}

To verify the validity of the proposed architecture, we show the results of computer simulation for 
system identification. The unknown system is a $10-$ taps lowpass FIR filter with delay 16, whose frequency response is defined as follows:

$$
H\left(e^{j w}\right)=\left\{\begin{array}{cc}
e^{-j w(N-1) / 2}, & 0 \leq|w| \leq \pi \\
0, & \text { otherwise }
\end{array}\right.
$$

Transversal filter containing 32-taps and using stepsize equal to 0.001 is the identified structure performed with $N L M S, D N L M S$, while the input sequence is Gaussian distribution, zero-mean random process. The implementation of adaptive noise cancellation scheme and adaptive $D L M S$ filter are shown in Fig. 4. The detail design of connected $F D A F$ without delay effect is shown in Fig. 5. The performance of error is shown in Fig. 6.

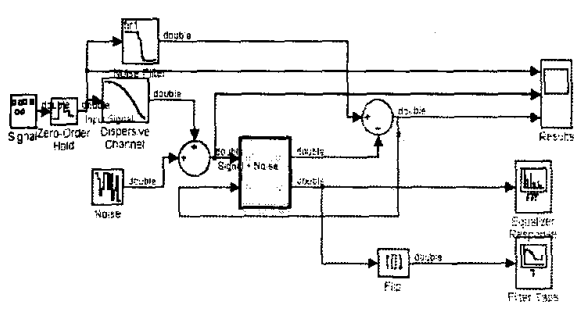

(a) Adaptive Noise Cancellation Scheme

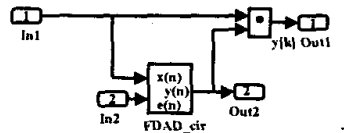

(b) Detail Design of DLMS filter in Frequency domain

Fig. 4. Implementation of Adaptive DLMS Filter

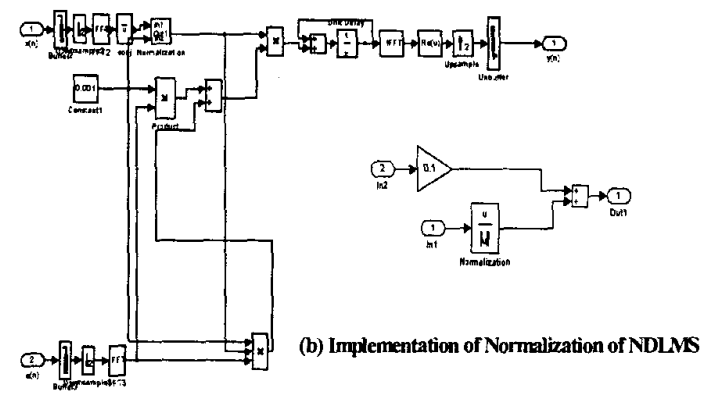

(a) Biock Diagram of Architecture based on the CDIMS

Fig. 5. Detail design of corrected FDAF without delay effect.

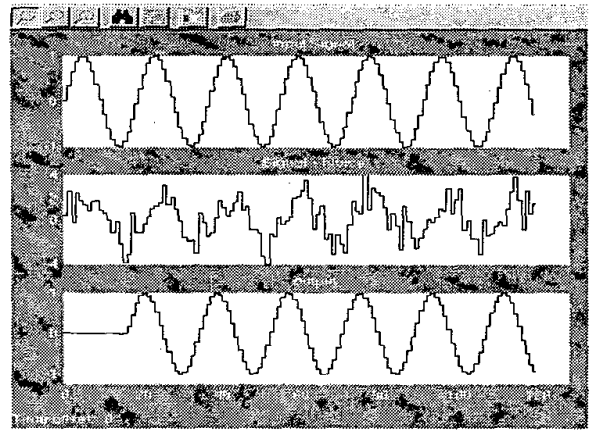

Fig. 6 The output of the proposed FDAD architecture.

In Fig. 6, the performance of the proposed architecture is equivalent to that of the NLMS. The detail circuit of realization the proposed PcDLMS is shown in Fig. 7.

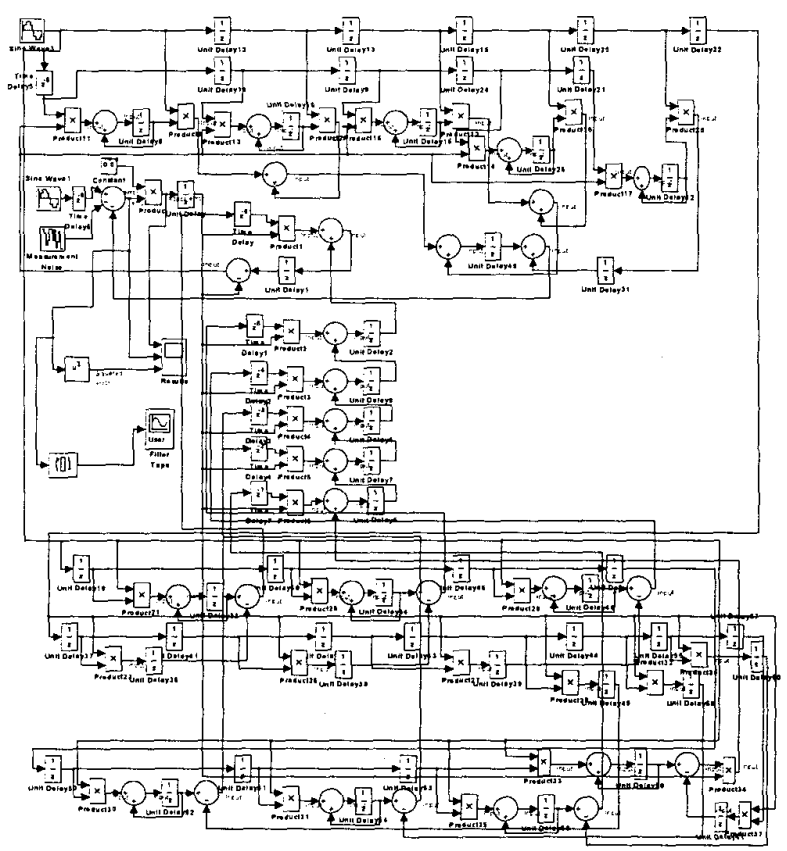

Fig. 7. The detail circuit of realization the proposed $P c D L M S$ Adaptive Filter

\section{CONCLUSIONS}

In this paper, we propose a new architecture for $D L M S$ algorithm in frequency-domain. This architecture enables us to simultaneously achieve 
good convergence characteristics, short latency and high throughput simultaneously without adaptation delays. To verify the validity of the proposed architecture, we show the results of the computer simulation of system identification by Simulink [Matlab5.3] and compare the characteristics of the proposed architecture with the conventional ones. In addition, it remains the advantage of the NLMS i.e., the step size assures that the convergence rate is determined automatically.

\section{References}

[1] R. D. Poltamnn, "Conversion of delayed LMS algorithm into the LMS algorithms," IEEE Signal Processing Lett., vol. 2, p.223, Dec. 1995.

[2] Q. Zhu, S. C. Douglas, and K. F. Smith, "A Pipelined Architecture for LMS Adaptive Filters Without Adaptation Delay," Proc. IEEE ISCAS '97, pp. 1933 1936, Jun. 1997.

[3] K. Matsubara, K. Nishikawa, and H. Kiya, "Pipelined LMS adaptive filter using a new look-ahead transformation," Proc. IEEE ISCAS '97, pp. 1933-1936, 1997.

[4] A. Harada, K. Nishikawa, H. Kiya, "A Pipelined Architecture for Normalized LMS Adaptive Digital Filters," IEICE, Trans. Fundamentals, vol. Es2-A, No. 2, pp. 223-229, Feb., 1999

[5] T. Kimijima, K. Nishikawa, and H. Kiya "An Effective Architecture of the Pipelined LMS Adaptive Filters," IEICE Trans. Fundamentals, vol. E82-A, no. 8, pp. 1428-1434, Aug. 1999.

[6] J. J. Shynk, "Frequency-Domain and Multirate Adaptive Filtering," IEEE, Signal Processing Magazine, pp.14-37, Jan. 1992.

[7] G. A. Clark, S. R. Parker, and S. K. Mitra, "A unified approach to time- and frequency- domain realization of FIR adaptive digital filters," IEEE, Trans. on Acoust. Speech, Sig. Proc., vol. ASSP-31, no. 5, pp. 1073-1083, Oct. 1983.

[8] M. Dentino, J. M. McCool, and B. Widrow, "Adaptive filtering in the frequency domain," Proc. IEEE, vol. 66, no. 12, pp. 1658-1659, Dec. 1978.

[9] S. S. Narayan, A. M. Peterson, and M. J. Narasimha, "Transform domain LMS algorithm," IEEE, Trans. on Acoust. Speech, Signal Processing, vol. 31, pp. 609-615, June 1983. 